\section{Hematoma retroperitoneal con neuropatía del femoral, ¿actitud conservadora o quirúrgica?}

\section{Sr. Director:}

Cada día se incrementa más el número de pacientes anticoagulados debido al aumento de la expectativa de vida y los beneficios demostrados de la terapia anticoagulante en patologías como síndrome coronario agudo, fibrilación auricular y enfermedad tromboembólica (1). Las hemorragias más frecuentes en pacientes anticoagulados ocurren en tracto gastro-intestinal, tejidos blandos y tracto urinario (2), siendo el sangrado en retroperitoneo una complicación rara pero conocida que requiere un alto índice de sospecha (1). Los factores que aumentan el riesgo de sangrado son la edad avanzada, las enfermedades concomitantes, los fármacos asociados y la intensidad de la anticoagulación (2). El hematoma retroperitoneal espontáneo (HRE) es una complicación poco frecuente pero importante, ya que puede producir alteraciones hemodinámicas graves y secuelas neurológicas severas (3). Presentamos un caso de hematoma espontáneo afectando a músculo psoas-ilíaco en una paciente anticoagulada con enoxaparina ( $1 \mathrm{mg} / \mathrm{kg} / 12 \mathrm{~h})$, que cursó con neuropatía del femoral como complicación, haciendo hincapié en que el tratamiento quirúrgico urgente es de elección cuando existe síndrome compartimental con afectación isquémica del nervio femoral $(4,5)$.

Mujer de 78 años que acude a Urgencias por cuadro clínico de 1 semana de evolución de glucemias capilares elevadas, disminución del nivel de conciencia, y tos seca y dificultad respiratoria en las últimas horas. Entre sus antecedentes destacaba diabetes mellitus a tratamiento insulínico con mal cumplimiento, hipertensión arterial y cardiopatía isquémica. En la exploración se objetivó temperatura de $38,8^{\circ} \mathrm{C}$ y 35 respiraciones por minuto. En la analítica destacaba una $\mathrm{PO}_{2}$ de $58 \mathrm{~mm} / \mathrm{Hg}$ con $\mathrm{SO}_{2}$ de 90,2\% con hemograma, bioquímica y coagulación normales. Ante sospecha de embolismo pulmonar se realiza gammagrafía de ventilación/perfusión en la cual se observan defectos subsegmentarios en lóbulo inferior izquierdo, lóbulo medio derecho y lóbulo inferior derecho con menor afectación ventilatoria, sugestivos de embolismo pulmonar en fase evolutiva o de revascularización. Se inicia tratamiento con enoxaparina $(1 \mathrm{mg} / \mathrm{kg} / 12 \mathrm{~h})$ y siete días después inicia cuadro súbito de dolor en miembros inferiores, región lumbar y cadera izquierda, con sudoración, palidez e hipotensión, hipoestesia y parestesias en miembros inferiores; junto a hemoglobina 7,2 $\mathrm{mg} / \mathrm{dl}$, hematocrito $23,3 \%$, tiempo de protrombina 16,2 seg, INR 1,24, TTPA 35,3 seg, glucosa $606 \mathrm{mg} / \mathrm{dl}$, urea $83 \mathrm{mg} / \mathrm{dl}$, creatinina 3,5 mg/dl, Na $129 \mathrm{mEq} / \mathrm{dl}$ y K $5,9 \mathrm{mEq} / \mathrm{dl}$. Se realiza tomografía computarizada evidenciándose gran hematoma retroperitoneal (Fig. 1), por lo que se consulta al servicio de Urología que en principio descarta tratamiento quirúrgico, recibiendo tratamiento con sueroterapia, analgesia, transfusión de hematíes y reversión de anticoagulación. A pesar de estas medidas continúa el dolor, parestesias y aparece plejía de miembro inferior izquierdo con hiporreflexia. Ante la afectación de sistema nervioso periférico confirmada con estudio electromiográfico (denervación completa de la musculatura dependiente del N. femoral), se decide intervención quirúrgica. Se realiza drenaje de hematoma retroperitoneal por laparotomía 15 días después del inicio del cuadro. La cirugía fue seguida de un inicio lento de movilización de miembro inferior y desaparición absoluta del dolor y las parestesias.

La etiología más frecuente del HRE es la rotura de un aneurisma de aorta abdominal (6). Cuando se produce en un paciente anticoagulado no suele existir lesión estructural causante y se suele presentar como un shock hipovolémico (dolor abdominal e inguinal, hipotensión y descenso del hematocrito) (1). La neuropatía del femoral se produce como consecuencia del aumento de la presión a nivel de un compartimento cerrado. La clínica de sospecha es dolor en región inguinal y cara interna de muslo, parestesias locales, hipoestesia o anestesia en cara antero-interna de

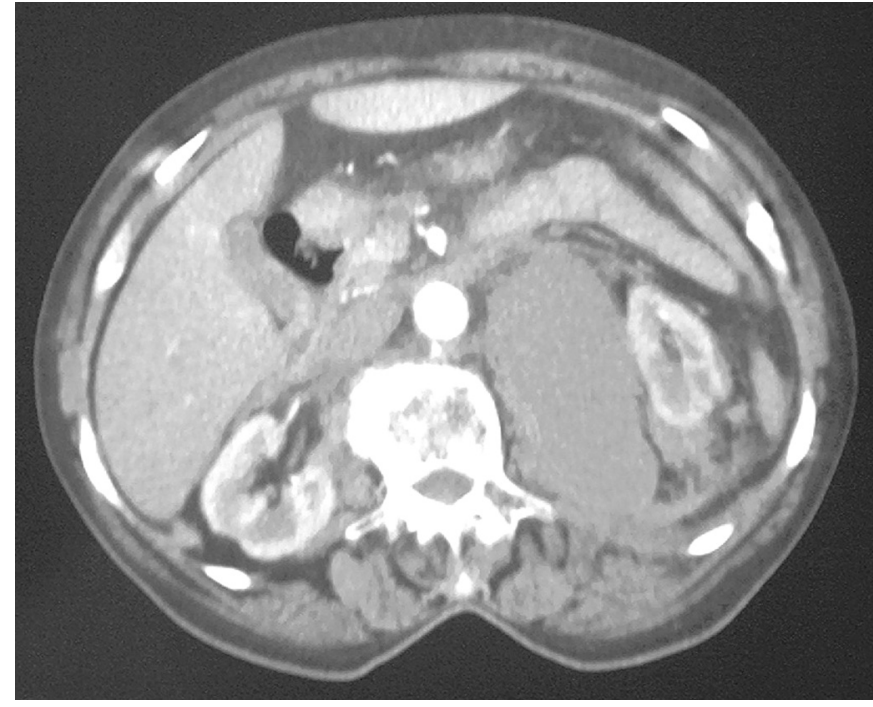

Fig. 1. TC con gran hematoma retroperitoneal izquierdo.

muslo y parálisis flácida con debilidad para la flexión de la cadera e impotencia para extensión de la rodilla (7). El tratamiento del hematoma retroperitoneal suele ser conservador con analgesia, sueroterapia, transfusiones y reversión de la anticoagulación (6) Los últimos estudios publicados indican la necesidad de intervención quirúrgica si existe compresión sobre vena cava inferior, riñón, uréter o nervio femoral (1). Nosotros apreciamos en nuestro caso una recuperación rápida y buena tras la intervención quirúrgica. La descompresión percutánea es otra alternativa ya que resuelve inmediatamente el dolor y provoca efectos beneficiosos en la recuperación de la función del nervio femoral, obviando la cirugía (8). El retraso en la resolución del cuadro compartimental y descompresión del nervio femoral conlleva una incompleta recuperación funcional, por lo que nosotros también aconsejamos actuación inmediata en estos casos.

\section{A. B. Argibay Filgueira, B. Maure Noia, P. Lamas Domín- guez, C. Martínez-Vázquez}

Servicio de Medicina Interna. Complejo Hospitalario Universitario. Vigo, Pontevedra

1. González C, Penado S, Llata L, Valero C, Riancho JA. The clinical spectrum of retroperitoneal hematoma in anticoagulated pacients. Medicine 2003; 82: 257-262

2. Landefeld CS, Beyth RJ. Anticoagulant related bleeding: clinical, epide miology, prediction and prevention. Am J Med 1993; 95: 315-328.

3. Horcajadas A, Abdullah O, Ros B, Katati MJ, Arráez MA, y Castañeda M. Neuropatía femoral secundaria a hematoma en el músculo ilíaco: a propósito de un caso. Neurocirugía 2000; 11: 450-454.

4. Civera Muñoz JE, Pérez Folques JE, Guirao Manzano J, Vázquez Ruiz J, Mansilla Molina D, Hernández Martínez J. Neuropatía femoral secundaria a hematoma espontaneo del músculo psoasilíaco. Cir Esp 1999; 65: 248-251

5. Montoya JP, Pokala N, Melde SL. Retroperitoneal hematoma and enoxaparin. Ann Intern Med 1999; 131: 796-797.

6. Pode D, Caine M. Spontaneous retroperitoneal hemorrhage. J Urology 1992; 147: 311-8

7. Acea Nebril B, Tabeada Filgueira L, Sánchez González F, Freire Rodríguez D, Fraguela Marina J, Aguirrezabalaga González J, et al. Abdomen agudo en pacientes anticoagulados. Valoración e indicaciones quirúrgicas. Rev Clin Esp 1995; 195: 463-467.

8. Holscher RS, Leyten FS, Oudenhoven LF, Puylaert JB. Percutaneous descompresion of an iliopsoas hematoma. Abdom Imaging 1997; 22 114-116. 